\title{
Case Report \\ Recurrent Syncope in a Cancer Patient: A Case Report and Review of the Literature
}

\author{
Jyoti Sharma and Anne H. Dougherty \\ Division of Cardiology, Department of Medicine, University of Texas at Houston, 6431 Fannin Street MSB 1.246 Houston, \\ TX 77054, USA \\ Correspondence should be addressed to Jyoti Sharma, jsharma8@gmail.com
}

Received 15 October 2010; Revised 3 January 2011; Accepted 24 January 2011

Academic Editor: Syed Wamique Yusuf

Copyright (๑) $2011 \mathrm{~J}$. Sharma and A. H. Dougherty. This is an open access article distributed under the Creative Commons Attribution License, which permits unrestricted use, distribution, and reproduction in any medium, provided the original work is properly cited.

A 59-year-old male with invasive squamous cell carcinoma of the left preauricular region, treated with several chemotherapy regimens and radiation therapy, was admitted for recurrent syncopal episodes. He was found to be suffering from neurocardiogenic reflex-mediated syncope secondary to mechanical compression of the carotid baroreceptors and glossopharyngeal nerve by the tumor. We discuss the pathophysiology of this case and the available treatment options.

\section{Case}

A 59-year-old Caucasian male with invasive squamous cell carcinoma of the left pre-auricular region was admitted for recurrent syncope over a period of four weeks. He was previously treated with radiation therapy and two chemotherapy agents, Cisplatin and Cetuximab. He described brief prodromal symptoms of lightheadedness and diaphoresis prior to each episode. He regained consciousness in less than one minute and quickly returned to his baseline mental status. Upon arrival to the emergency room after his first episode, his pulse was 65 beats per minute (bpm) and his blood pressure was recorded as $64 / 42 \mathrm{~mm} \mathrm{Hg}$. He was managed with aggressive fluid resuscitation, including three liters of intravenous fluids, and Trendelenburg positioning. After this first episode, follow-up blood pressure recordings were in the normal range within the first one to two hours. 24 hours later, he was no longer symptomatic and was back to his baseline physical and mental status. On a second occasion he was more profoundly affected, and his first systolic blood pressure recorded in the emergency room was $46 \mathrm{~mm} \mathrm{Hg}$ and his pulse ranged from $46-57 \mathrm{bpm}$. Upon presentation during this event he was confused and his blood pressure took longer to recover despite similar treatment with intravenous fluid resuscitation and Trendelenburg positioning. However, similar to the first episode, 24 hours later he was back to his baseline.

On no occasion was his syncope associated with head turning or neck compression. He did have longstanding neuropathic pain located in the posterior nasopharynx and the left side of his face. As his disease had progressed, he also developed pain with swallowing; however, there was no exacerbation in his symptoms surrounding the syncopal episodes. Several events occurred while he was supine.

Further testing including orthostatic blood pressure measurements, electrolyte measurements, an electrocardiogram, and echocardiogram revealed no abnormalities. A Holter monitor recording preceding one of his syncopal episodes showed sinus pauses of up to 2.1 seconds. These pauses were frequent and were associated with prodromal symptoms of diaphoresis and lightheadedness (Figure 1(a)). A few minutes later, he had a rapid drop in his sinus rate to a junctional rhythm at $33 \mathrm{bpm}$ (Figure 1(b)) that culminated in a syncopal episode. Both of these symptomatic recordings, although nocturnal, began in the supine, but awake state. Formal tilt table testing and gentle carotid massage was not feasible due to the lability of his vital signs. Repeat head and neck computed tomography (CT) scan showed progression of his tumor, now completely encasing his left internal carotid artery at its bifurcation from the left common 


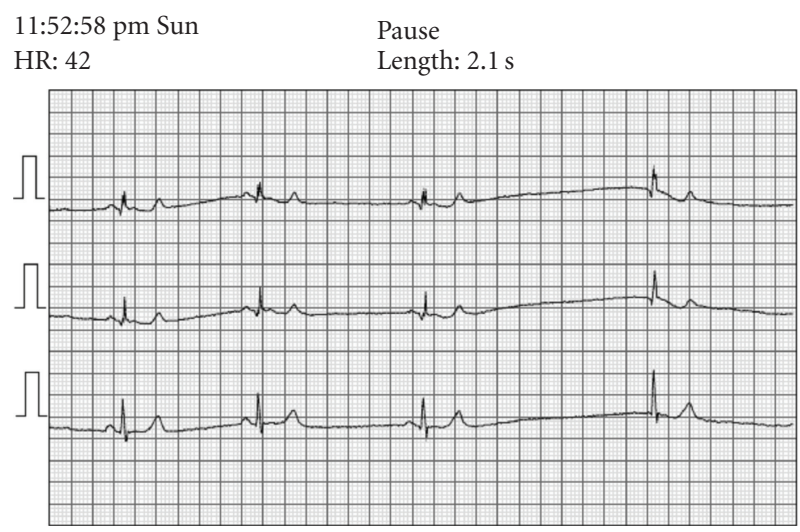

(a)

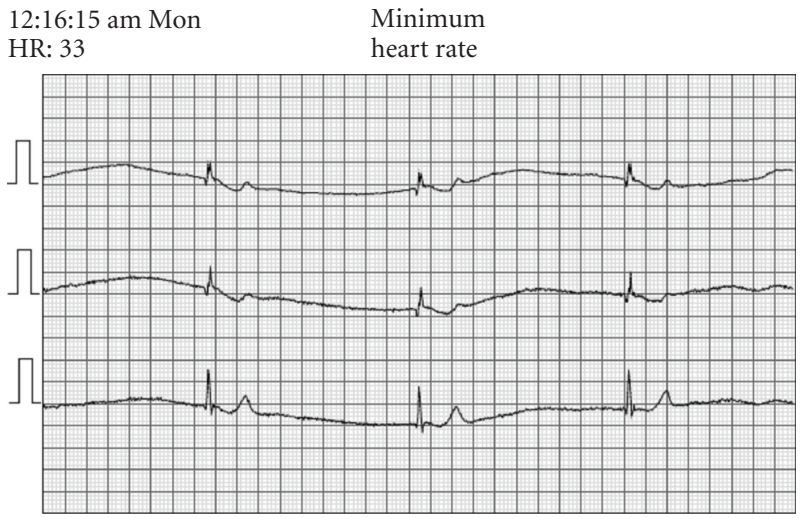

(b)

FIgURE 1: (a) Holter monitor recording showing sinus bradycardia at $42 \mathrm{bpm}$ with sinus pauses of up to 2.1 seconds, corresponding to his prodromal symptoms while awake. (b) Holter monitor recording during a syncopal episode showing a drop in heart rate to 33 bpm.

carotid artery (Figure 2). In addition, the tumor abutted the glossopharyngeal nerve at the point of exit from the posterior aspect of the base of his skull. We concluded that the patient was suffering from neurocardiogenic reflex-mediated syncope, secondary to mechanical compression by the tumor of the carotid baroreceptors and the glossopharyngeal nerve. This syndrome has also been called a parapharyngeal space lesion syncope.

He was placed on midodrine, fludrocortisone, and sertraline to help alleviate his symptoms; however, he continued to have syncopal episodes while hospitalized that became progressively more resistant to fluid resuscitation. For example, during the first two events he experienced, his blood pressure recovered within the first one to two hours of aggressive fluid resuscitation. However, during later hospitalizations, despite the same interventions his blood pressure remained in the hypotensive range for hours. During two different episodes, he was given atropine to help increase his heart rate. His heart rate increased appropriately with this intervention; however, hypotension persisted, thus demonstrating that the vasodilatory component of his neurally mediated syncope was playing a dominant role in his events. His primary oncology team determined that he had a poor prognosis and did not have any more chemotherapy or radiation therapy options available to treat his progressive cancer. After discussion with him, he was discharged with home hospice.

\section{Discussion}

2.1. Neurally Mediated (Reflex) Syncope and Carotid Baroreceptors. Neurally mediated, or reflex, syncope is the term used to describe a reflex response that leads to systemic hypotension and bradycardia. The trigger for this response can be varied in individual patients. This case illustrates a particular severe cause of neurally mediated syncope due to mechanical encroachment on the carotid baroreceptors and glossopharyngeal nerve.

The carotid baroreceptors are located at the internal carotid artery, close to their bifurcation off the common

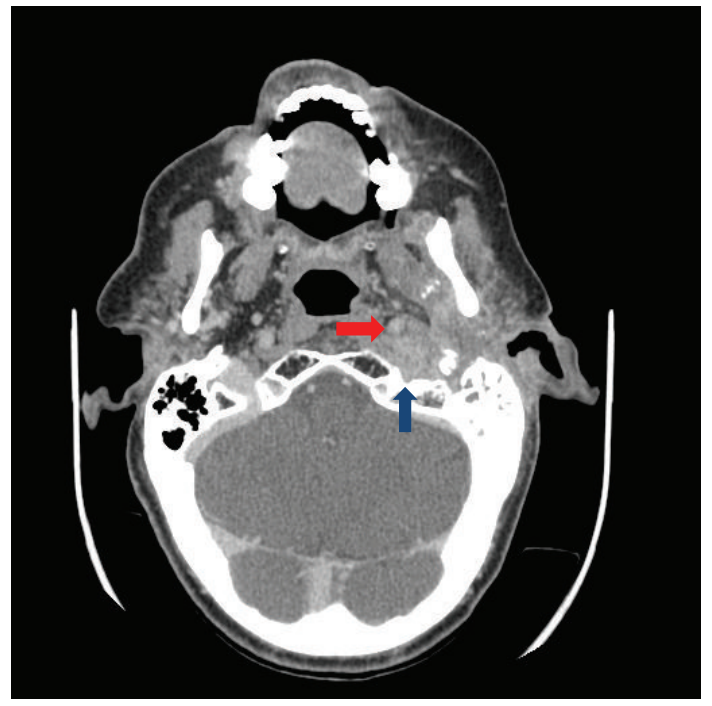

Figure 2: CT scan of the patient's head, axial image. The red arrow shows tumor encasing the internal carotid artery. The blue arrow shows tumor infiltrating the skull base where CN IX exits the jugular foramen. CN IX is not visualized clearly because of the invading tumor.

carotid artery. They are innervated by the Sinus Nerve of Hering, which is a branch of the glossopharyngeal nerve, cranial nerve $(\mathrm{CN})$ IX. Sensory fibers arise from the carotid body and join the other components of CN IX at the inferior hypoglossal ganglion. The central processes of these neurons enter the skull via the jugular foramen. In its passage to the jugular foramen, CN IX passes between the internal jugular vein and internal carotid artery. It descends in front of the internal carotid artery and beneath the styloid process and the muscles connected with it. After entering the skull, CN IX eventually synapses in the nucleus tractus solitarus (NTS), which in turn modulates activity of both the sympathetic and parasympathetic neurons in medulla. 
The medulla ultimately regulates autonomic control of the heart and blood vessels.

Maximal carotid sinus sensitivity normally occurs near a mean arterial pressure (MAP) of approximately $100 \mathrm{~mm} \mathrm{Hg}$. Increases in MAP increase baroreceptor firing and decreases in MAP lead to decreases in baroreceptor firing. Carotid sinus sensitivity is such that even very small changes in arterial pressure around this set point can significantly alter receptor firing, and in turn, tightly up regulate or down regulate autonomic control of the heart and peripheral vasculature [1]. In this individual, progressive disease led to tumor compression of the internal carotid artery, and the mechanical pressure caused an increase in baroreceptor firing. His medulla responded by increasing parasympathetic outflow and decreasing sympathetic outflow. Under normal physiologic conditions, baroreceptor firing has a tonic inhibitory influence on sympathetic outflow from the medulla, and in this case, the sympathetic outflow was decreased still further.

This patient's tumor also encroached upon CN IX as it exited the skull base and thus affected a second anatomical component of the neutrally mediated reflex. Direct tumor ingrowth at CN IX at the jugular foramen interrupted the sensory fibers that run along the nerve affecting parasympathetic and sympathetic outflow from the medulla. The combination of bradycardia and peripheral vasculature vasodilatation that occurs as a result of the surge in parasympathetic outflow and decrease in sympathetic stimulation culminated in his syncopal episodes.

Another pathogenic mechanism underlying the association between glossopharyngeal neuralgia and syncope is thought to be related to the close relationship that exists between CN IX and CN X [2]. Neurosurgical research suggests that ephapses, or artificial synapses, develop between $\mathrm{CN}$ IX and CN X, or that anomalous communications develop between the nucleus tractus sollitarus and the nucleus ambiguous centrally [3]. The presence of an irritative lesion might thus give rise to "cross talk" between the above circuits, thereby inducing bradycardia with or without a decrease in peripheral resistance, through possible mediation of the vagus nerve and carotid baroreceptor, respectively [4]. Evidence for cross talk between nerve fibers is supported by experimental findings [5].

Thus, it is likely a combination of several factors including direct tumor invasion on the carotid body and CN IX, and possible development of anomalous connections centrally involving $\mathrm{CN} X$ that led to our patient's syncopal episodes. The inexorable progression of his symptoms and their increasing refractoriness to resuscitation corresponds to the progressive state of his tumor.

2.2. Treatment Options for Neurally Mediated (Reflex) Syncope. The European Society for Cardiology offered a recent update on its guidelines for the management of syncope [6]. The initial steps for treating patients suffering from the common form of neurally mediated (reflex) syncope include reassurance and education about their syndrome. Other simple interventions such as educating patients to avoid trigger events, teaching them to recognize and respond to premonitory symptoms, and modifying or discontinuing medications that may cause hypotension have also proven to be beneficial. Patients that suffer from frequent syncopal episodes may require additional pharmacologic treatment.

Many drugs have been used for treatment of the various forms of neurally mediated syncope, including beta blockers and anticholinergic agents; however, the evidence for benefit in randomized clinical trials is lacking. Midodrine, an agent that stimulates alpha-1 adrenergic receptors, has shown more promise in selected patients, particularly those with predominant vasodilatory responses. Results from two small open-label randomized studies that were done on patients who suffered from recurrent neurally mediated syncope showed that midodrine was effective in reducing the number of syncopal and presyncopal episodes over the short term, and its use significantly improved patients' quality of life $[7,8]$.

The use of selective serotonin reuptake inhibitors (SSRI) has also been studied in patients with neurally mediated syncope. Serotonin (5-hydroxytryptamine), along with several other neurotransmitters, may play an important role in eliciting reflex syncopal reactions. Experimental animal models in rats [9] have shown that intracerebral serotonin inhibits sympathetic neural outflow during acute hemorrhage while increasing adrenal sympathetic stimulation. In rabbits, blocking serotonin receptors seemed to eliminate the hypotensive reflex during acute hemorrhage [10]. Accumulation of extracellular serotonin by SSRIs result in downregulation of neuronal transmission, thus blunting the effect of the neurocardiogenic reflex.

Paroxetine was studied in a randomized, double-blind, placebo-controlled trial in patients who suffered from recurrent vasovagal syncope (VVS) [11]. 68 consecutive patients were included and those that received paroxetine were significantly less likely to suffer from spontaneous syncope (17.6\% in the paroxetine group compared to $52.9 \%$ in the placebo group) over a mean follow-up period of 25.4 months. Similar results were reported in studies evaluating other SSRIs including fluoxetine [12] and sertraline [13]. Despite success in these small trials, until their results can be duplicated in larger trials, the European Society of Cardiology is not currently recommending use of this class of agents.

Besides pharmacologic therapy, much effort has been devoted to examining whether pacemaker insertion is useful in treating various forms of neurally mediated syncope, and in particular vasovagal syncope. Five major multicenter randomized controlled trials have been done so far to evaluate the benefit of pacing in this patient population [14-18].

The first three studies [14-16] published showed a significant benefit to patients who were implanted with a pacemaker. The two most recent studies, however, showed a negative result. The largest difference in these studies was the method in which they were conducted. The initial three studies, theVPS Study [14], European VASIS trial [15] and SYDIT trial [16] were all unblinded studies. Patients were randomized to receive either a pacemaker or standard 
medical therapy. In the latter two studies, the SYNPACE trial [17] and the VPS II study [18], all patients received a pacemaker, but patients were randomized to have their device either turned on or turned off.

Combining the data of these five trials, only 318 patients are studied. In addition, because the first three trials were unblinded, placebo effect and reporting bias must be taken into consideration when examining the results of these studies. While pacing may be efficacious for the bradycardic component of neurally mediated syncope, it does little to combat the accompanying hypotension. Thus, given the evidence, pacing therapy is not considered first line therapy for most patients with neurally mediated syncope, and the European guidelines state "cardiac pacing should be limited as a choice of last resort to a very selected small proportion of patients affected by severe vasovagal syncope [6]." In the subject of this case report, the vasodepressor component of his syncope overshadowed the cardioinhibitory component, thus pacing was not considered to be a reasonable treatment choice for this individual.

\subsection{Treatment of Syncope in Cancer Patients. Recurrent} carotid sinus syncope in head and neck cancer patients is uncommon but can present a particularly malignant form of the condition. The presumed mechanism is direct tumor compression of the carotid sinus baroreceptors or CN IX, or both, as in the case above. There are no guidelines currently in place to direct the treatment for syncope in this specific patient population. Several case reports have been published with circumstances similar to those seen in our patient. Most recently, two groups reported resolution of syncopal episodes with tumor regression with chemotherapy $[19,20]$. In one case, the patient had a pacemaker placed but continued to faint. However, in both cases symptoms resolved after induction chemotherapy was begun.

Older published reports have also documented similar situations. One case report examined a group of 17 patients suffering from head and neck carcinoma with associated syncope [21]. Various treatment strategies including carotid ligation, intracranial sectioning of CN IX, and local radiation have been employed with success in individual reports. Of the 17 patients, three had pacemakers placed and this appeared to be ineffective in preventing syncope. Another report with seven patients found periarteriectomy, intracranial nerve root section, and radiation therapy to be beneficial [22].

A review from the neurosurgery literature examines treating CN IX dysfunction by surgical means. A group from France investigated patients who were suffering from syncopal episodes secondary to tumor compression of CN IX [23]. The majority of these patients were treated with neurosurgical resection of the root of CN IX, though not all surgeries were completely curative. In addition to discussing surgical options for these types of patients, the authors also discuss the importance of relief of symptoms with treatment of the underlying cause-invading tumor.
What many of these successfully treated cases have in common is achievement of regression of the underlying carcinoma, reduction in tumor burden, and relief of pressure on either the carotid baroreceptors, CN IX, or both. Pacemaker implantation has been an unsuccessful treatment strategy in this group of patients, due to a pacemaker's inability to combat the vasodilatory component of neurally mediated syncope. Other medical treatment options, like those tried in this patient, are often left to the discretion of the treating physician because there are no current treatment guidelines available. Definitive treatment and successful syncope relief depends primarily on successful tumor regression, without which the condition is likely to be inexorably disabling and perhaps fatal.

\section{References}

[1] R. Klabunde, Cardiovascular Physiology Concepts, Lippincott Williams \& Wilkins, Philadelphia, Pa, USA, 2005.

[2] L. Ferrante, M. Artico, B. Nardacci et al., "Glossopharyngeal neuralgia with cardiac syncope," Neurosurgery, vol. 36, no. 1, pp. 58-63, 1995.

[3] W. J. Gardner, "Concerning the mechanism of trigeminal neuralgia and hemifacial spasm," Journal of neurosurgery, vol. 19, pp. 947-958, 1962.

[4] K. Reddy, D. E. Hobson, A. Gomori, and G. R. Sutherland, "Painless glossopharyngeal 'neuralgia' with syncope: a case report and literature review," Neurosurgery, vol. 21, no. 6, pp. 916-919, 1987.

[5] A. S. Marazzi and R. Lorente De No, "Interaction of neighboring fibres in myelinated nerve," Journal of Neurophysiology, vol. 7, pp. 83-100, 1944.

[6] "Guidelines on Management (Diagnosis and Treatment) of Syncope-update 2004," European Heart Journal, vol. 25, pp. 2054-2072, 2004.

[7] C. R. Ward, J. C. Gray, J. J. Gilroy, and R. A. Kenny, "Midodrine: a role in the management of neurocardiogenic syncope," Heart, vol. 79, no. 1, pp. 45-49, 1998.

[8] A. Perez-Lugones, R. Schweikert, S. Pavia et al., "Usefulness of midodrine in patients with severely symptomatic neurocardiogenic syncope: a randomized control study," Journal of Cardiovascular Electrophysiology, vol. 12, no. 8, pp. 935-938, 2001.

[9] D. A. Morgan, P. Thoren, E. A. Wilczynski, R. G. Victor, and A. L. Mark, "Serotonergic mechanisms mediate renal sympathoinhibition during severe hemorrhage in rats," American Journal of Physiology, vol. 255, no. 3, pp. 496-502, 1988.

[10] R. G. Evans, V. Kapoor, and J. Ludbrook, "A CNS serotonergic mechanism in acute central hypovolemia in conscious rabbits?" Journal of Cardiovascular Pharmacology, vol. 19, no. 6, pp. 1009-1017, 1992.

[11] E. D. Girolamo, C. D. Iorio, P. Sabatini, L. Leonzio, C. Barbone, and A. Barsotti, "Effects of paroxetine hydrochloride, a selective serotonin reuptake inhibitor, on refractory vasovagal syncope: a randomized, double-blind, placebo-controlled study," Journal of the American College of Cardiology, vol. 33, no. 5, pp. 1227-1230, 1999.

[12] B. P. Grubb, D. A. Wolfe, D. Samoil, P. Temesy-Armos, H. Hahn, and L. Elliott, "Usefulness of fluoxetine hydrochloride for prevention of resistant upright tilt induced syncope," Pacing and Clinical Electrophysiology, vol. 16, no. 3 I, pp. 458464, 1993. 
[13] B. P. Grubb, D. Samoil, D. Kosinski, K. Kip, and P. Brewster, "Use of sertraline hydrochloride in the treatment of refractory neurocardiogenic syncope in children and adolescents," Journal of the American College of Cardiology, vol. 24, no. 2, pp. 490-494, 1994.

[14] S. J. Connolly, R. Sheldon, R. S. Roberts, and M. Gent, "The North American vasovagal pacemaker study (vps) a randomized trial of permanent cardiac pacing for the prevention of vasovagal syncope," Journal of the American College of Cardiology, vol. 33, no. 1, pp. 16-20, 1999.

[15] R. Sutton, M. Brignole, C. Menozzi et al., "Dual-chamber pacing in the treatment of neurally mediated tilt-positive cardioinhibitory syncope: pacemaker versus no therapy: a multicenter randomized study," Circulation, vol. 102, no. 3, pp. 294-299, 2000.

[16] F. Ammirati, F. Colivicchi, and M. Santini, "Permanent cardiac pacing versus medical treatment for the prevention of recurrent vasovagal syncope: a multicenter, randomized, controlled trial," Circulation, vol. 104, no. 1, pp. 52-57, 2001.

[17] F. Giada, A. Raviele, C. Menozzi et al., “The vasovagal syncope and pacing trial (Synpace). A randomized placebo-controlled study of permanent pacing for treatment of recurrent vasovagal syncope," Pacing and Clinical Electrophysiology, vol. 26, p. 1016, 2003.

[18] S. J. Connolly, R. Sheldon, K. E. Thorpe et al., "Pacemaker therapy for prevention of syncope in patients with recurrent severe vasovagal syncope: second vasovagal pacemaker study (VPS 11): a randomized trial," Journal of the American Medical Association, vol. 289, no. 17, pp. 2224-2229, 2003.

[19] H. J. Ji, H. K. Myoung, G. K. Hoon et al., "Successful treatment of syncope with chemotherapy irresponsive to cardiac pacemaker in head and neck cancer," Yonsei Medical Journal, vol. 50, no. 5, pp. 725-728, 2009.

[20] Y. M. Choi, M. F. Mafee, and L. E. Feldman, "Successful treatment of syncope in head and neck cancer with induction chemotherapy," Journal of Clinical Oncology, vol. 24, no. 33, pp. 5332-5333, 2006.

[21] D. R. Macdonald, E. Strong, S. Nielsen, and J. B. Posner, "Syncope from head and neck cancer," Journal of NeuroOncology, vol. 1, no. 3, pp. 257-267, 1983.

[22] H. R. Muntz and P. G. Smith, "Carotid sinus hypersensitivity: a cause of syncope in patients with tumors of the head and neck," Laryngoscope, vol. 93, no. 10, pp. 1290-1293, 1983.

[23] X. Carrat, J. M. Francois, T. Houliat et al., "Syncopal neuralgia of the glossopharyngeal nerve caused by tumoral invasiveness," Revue de Laryngologie Otologie Rhinologie, vol. 117, no. 2, pp. 137-140, 1996. 


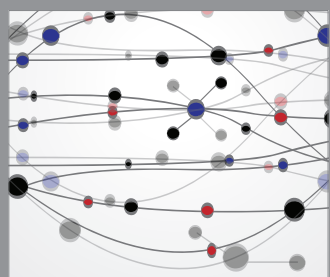

The Scientific World Journal
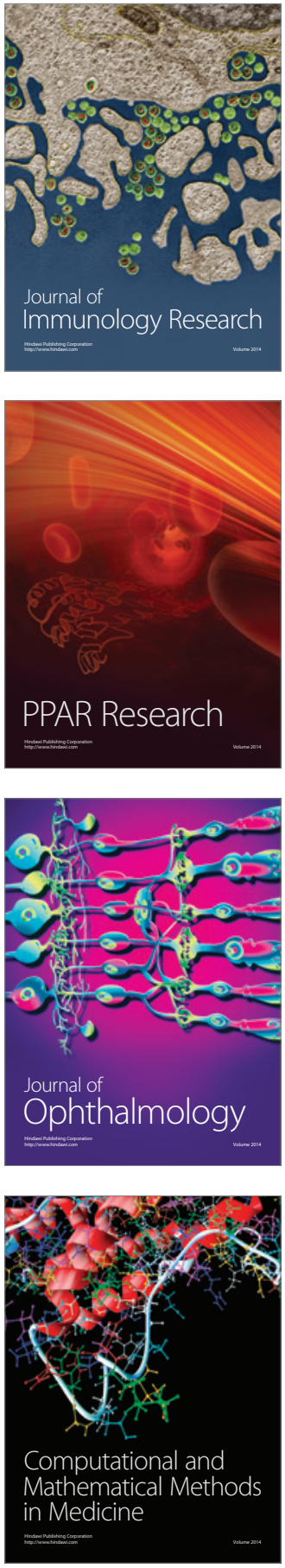

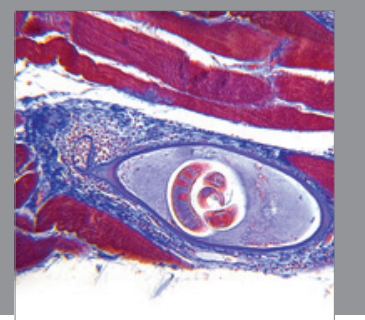

Gastroenterology

Research and Practice
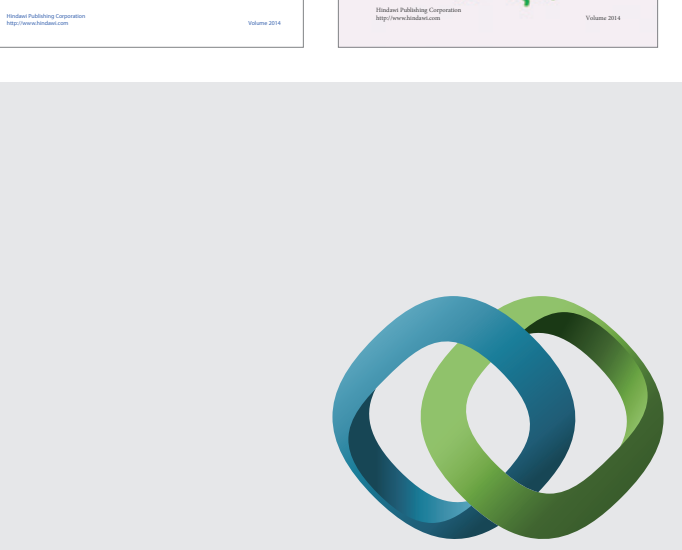

\section{Hindawi}

Submit your manuscripts at

http://www.hindawi.com
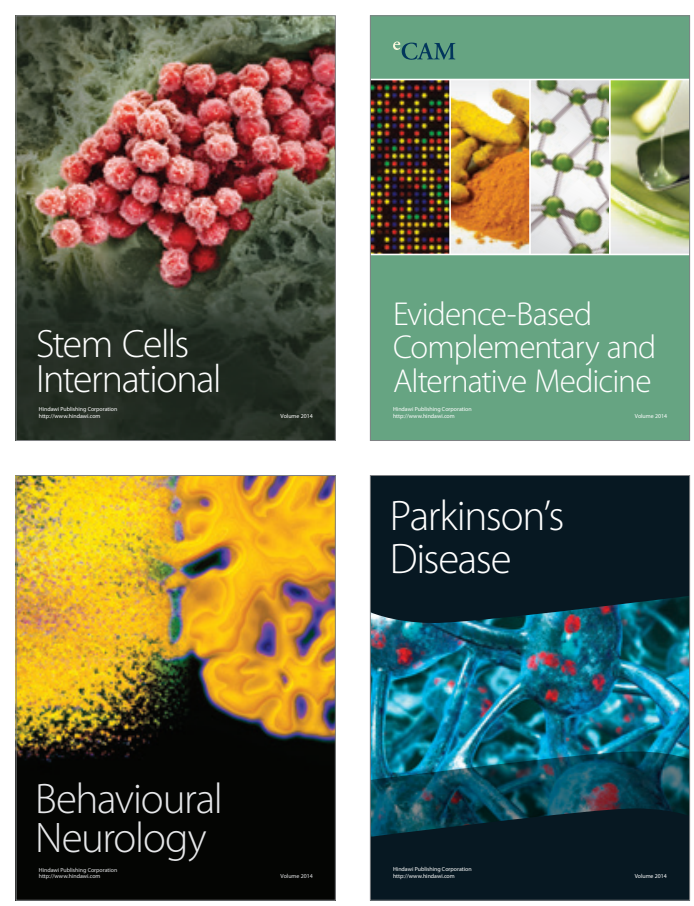

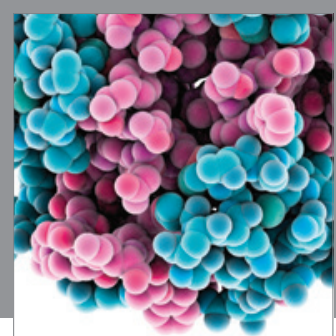

Journal of
Diabetes Research

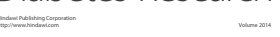

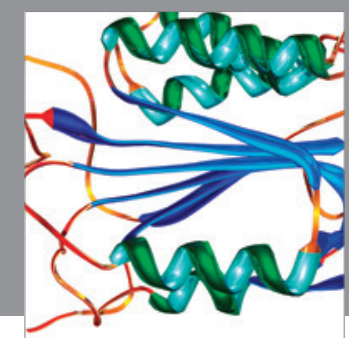

Disease Markers
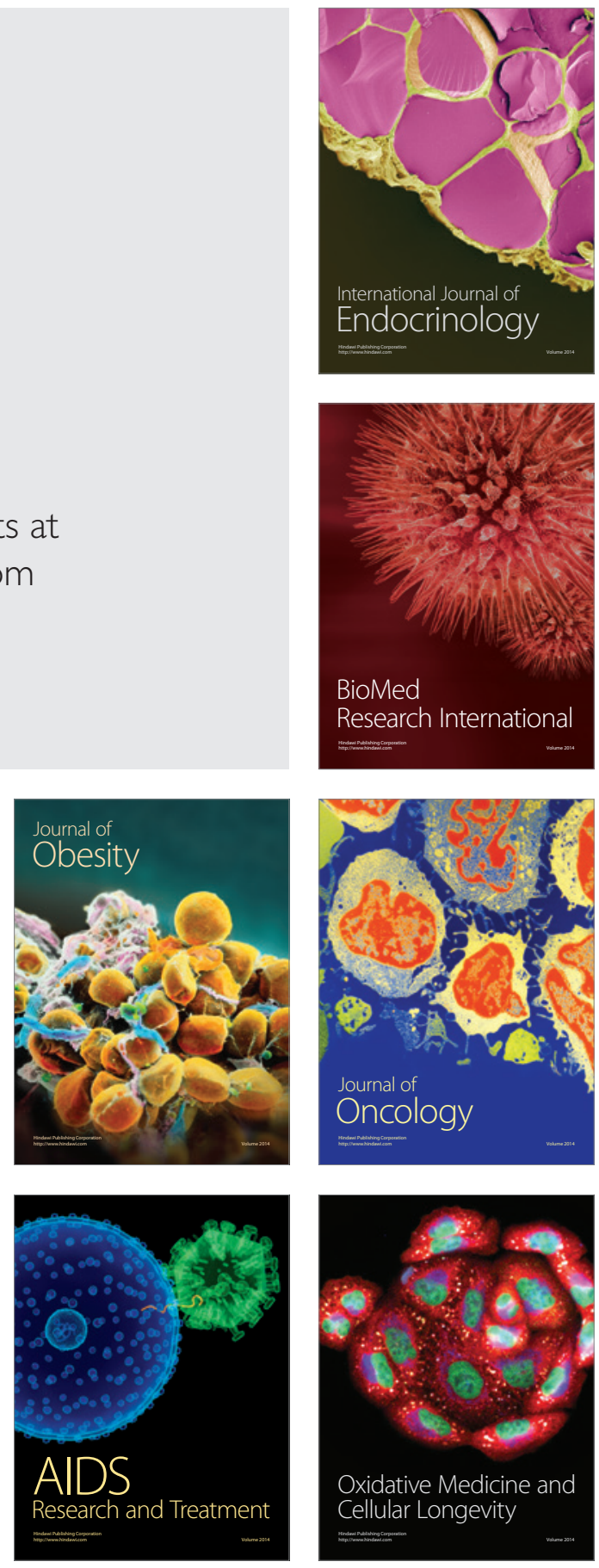